\title{
Elemental Sulfur Identified in Urine of Cheetah, Acinonyx jubatus
}

\author{
Ben V. Burger, Runine Visser, Alvira Moses \& Maritha Le Roux \\ Laboratory for Ecological Chemistry, Department of Chemistry, Stellenbosch University, Stellenbosch, 7600, South Africa
}

\begin{abstract}
The urine of the cheetah, Acinonyx jubatus, is almost odorless, and probably for this reason, it has not attracted much attention from scientists. Using gas chromatography-mass spectrometry, we identified 27 and 37 constituents in the headspace vapor of the urine of male and female cheetah, respectively. These constituents, composed of hydrocarbons, short-chain ethers, aldehydes, saturated and unsaturated cyclic and acyclic ketones, 2acetylfuran, dimethyl disulfide, dimethyl sulfone, phenol, myristic acid (tetradecanoic acid), urea, and elemental sulfur, are all present in the headspace vapor in very small quantities; dimethyl disulfide is present in such a low concentration that it cannot be detected by the human nose.

This is only the second example of elemental sulfur being secreted or excreted by an animal. It is hypothesized that the conversion of sulfur-containing compounds in the cheetah's diet to elemental sulfur and to practically odorless dimethyl sulfone enables this carnivore to operate as if "invisible" to the olfactory world of its predators as well as its prey, which would increase its chances of survival.
\end{abstract}

Key Words: Cheetah, Acinonyx jubatus, urine, territorial behaviour, mammalian semiochemicals, elemental sulphur.

\section{Introduction}

Although cheetah, Acinonyx jubatus, can reach a speed of more than $100 \mathrm{~km} / \mathrm{hr}$ in seconds (Caro, 1994), they are exposed to severe competition for survival because they lack the physical strength to guard their territory and protect their young against stronger predators. In some nature reserves, predation accounts for more than $40 \%$ mortality in cheetah litters (Eaton, 1974). Cheetah are territorial, mostly solitary, animals with enormous home ranges. The average size of the home range of a female on the Serengeti plains is about $830 \mathrm{~km}^{2}$. Male territories are about $4.5 \%$ of that of female territories (Caro, 1994).

Male and female cheetah leave territorial marks by spraying objects with urine. Females in heat squirt urine on bushes, tree trunks, and rocks to attract males (Macdonald, 1984), but marking behavior is more common in males. Consensus has not been reached as to the function of this behavior in males. Both sexes as well as cubs take great interest in marks left by a strange male, and there is convincing evidence suggesting that territorial marking is a mechanism whereby contact and conflict between family groups, which could result in injury or death, is avoided (Eaton, 1974).

Probably because the urine is almost odorless, it has not elicited much interest from scientists. Continuing our research on the semiochemicals of mammals (Burger, 2005), we attempted a comprehensive chemical characterization of the volatile fraction of the urine of the cheetah.

\section{Methods and Materials}

\section{Sample Collection}

Urine was collected from four captive male cheetah (Cheetah Outreach Facility, Spier Wine Estate, Stellenbosch, South Africa) by catching sprayed urine in a glass beaker. Bladder urine was obtained from an anesthetized female by catheterization. These cheetah were kept in enclosures varying in size from about $100 \times 50 \mathrm{~m}$ to $85 \times 70 \mathrm{~m}$ with wire mesh fences and were fed a diet of chicken. On one occasion, urine was collected from two wild males at the research station of the Cheetah Conservation Fund, Otjiwarongo, Namibia. The urine samples were frozen as quickly as possible and kept at $-25^{\circ} \mathrm{C}$ until used for analysis. 


\section{Analytical Techniques}

Volatile constituents of the urine were sampled by sorptive sample enrichment in polydimethylsiloxane (PDMS) rubber by using a sample enrichment probe (SEP) (Burger et al., unpublished data) followed by thermal desorption in the injector of a gas chromatography-mass spectrometry (GC-MS) instrument (GC-MS, Carlo Erba QMD 1000). The volatile organic constituents were also extracted from the urine with dichloromethane (Merck, Residue Analysis Grade) for retention-time comparison (co-elution) with authentic synthetic compounds and quantification of the elemental sulfur and some of the less volatile compounds extracted from the urine. The concentration of the sulfur in cheetah urine was determined by GC-MS with sulfur as external reference.

\section{Bioassays}

Thick, green paper sheets $(350 \times 230 \mathrm{~mm}$ ) were uniformly wetted on one side with $6 \mathrm{ml}$ of a colloidal sulfur solution $(3 \mu \mathrm{g} \mathrm{S} / \mathrm{ml}$ ) and air-dried to produce test papers coated on one side with $18 \mu \mathrm{g}$ of sulfur, equivalent to approximately $18 \mathrm{ml}$ of urine. Controls were prepared by wetting paper sheets with water, fresh cheetah urine, or with solutions of various odorants such as benzeneethanethiol and orange juice.

Bioassays were done at the Spier Cheetah Outreach Facility with four male and three female cheetah that were reasonably tame and accustomed to human visitors. Wet and dry sulfur-treated papers, wet and dry water-treated controls, and papers wetted with cheetah urine or with other odorants were presented $3 \mathrm{~m}$ apart along one of the fences of an enclosure where a cheetah was likely to spot and inspect it. The attention the cheetah paid to each test paper and control, as well as any peculiar behavior, was noted. Tests were also done at the Tygerberg Zoo with a breeding pair of wild cheetah, as well as a breeding pair each of the following species: lion (Panthera leo), Bengal tiger (P. tigris), caracal (Felis caracal), wild cat (F. silvestris lybica), and wild dog (Lycaon pictus).

\section{Results and Discussion}

Enrichment of the organic volatiles in the headspace of cheetah urine by using the SEP technique and GC-MS analysis of the enriched volatiles gave a total ion chromatogram (TIC) (Figure $\underline{1}$ ) and mass spectra that could be used for the identification of most of the headspace constituents. Urea (35) extracted from the headspace of the urine appears in the TIC as a broad peak at a retention time of $49.2 \mathrm{~min}$, and cyanuric acid (2,4,6-trihydroxy-1,3,5triazine) (39) as a broad peak at a retention time of $92.0 \mathrm{~min}$. This compound is formed when urea is heated to temperatures above its melting point, and it was concluded that its presence in the TIC shown in Figure $\underline{1}$ is due to the partial conversion of the urea to cyanuric acid in the injector of the GC-MS instrument.

The saddlelike elution profile between these two peaks shows that this process continued to take place in the column during the chromatographic process. Some of the compounds listed in Table $\underline{1}$ were detected in the headspace vapor of the urine of only one of the sexes. The dimethyl disulfide (9) is present in the urine in such a small quantity that it could not be detected by the human nose. We were surprised to find elemental sulfur in all of the urine samples from cheetah males and from one female cheetah. Sulfur is not detected by GC when a flame ionization detector (FID) is employed. However, the cyclic $S_{6}(37)$ and $S_{8}(40)$ forms of the element can be separated and quantified by GC-MS analysis. In the present investigation, small quantities of $\mathrm{S}_{6}$ and larger quantities of $\mathrm{S}_{8}$ were found in the headspace vapor and in dichloromethane extracts of the urine of both sexes.

Employing sulfur as external standard, it was found that the concentration of sulfur in the urine of male cheetah with unlimited access to water is approximately $1 \mu \mathrm{g} / \mathrm{ml}$. Sulfur is not an artifact formed from dimethyl disulfide because it was not detected when dimethyl disulfide was subjected to GC-MS analysis, either under the analytical conditions employed in the present investigation or under thermally harsher conditions. It was not possible to determine the concentration of the highly volatile constituents of the urine because they were largely lost when the extract was concentrated and could not be detected in GC and GC-MS analyses. The less volatile constituents, dimethyl sulfone (20), benzaldehyde (23), phenol (25), acetophenone (29), and 2-nonanone (30) were present in concentrations of about $250,1.5,0.6,1.4$, and $1.5 \mathrm{ng} / \mathrm{ml}$, respectively, in the extracted material. Although 2piperidone ( $\delta$-valerolactam) was not detectable in the urine headspace vapor, it was identified as the major organic constituent of cheetah urine at a concentration of $1.7 \mu \mathrm{g} / \mathrm{ml}$.

Several explanations can be offered for the presence of sulfur in cheetah urine. It is unlikely that this element could be a sex pheromone of the species because sulfur is present in the urine of both male and female cheetah. In view of the enormous home ranges of these animals, use of sulfur as a territorial-marking pheromone, or a component of such a pheromone, would have the advantage that it is much more persistent than the quite volatile 
organosulfur compounds present in the urine of other felids. It is also possible that the conversion of sulfurcontaining proteins to elemental sulfur is just an alternative mechanism by which sulfur is excreted in its elemental form instead of being excreted as organosulfur compounds, as in many other felids. The conversion of organosulfur compounds to sulfur and dimethyl sulfone, also a practically odorless compound, could allow cheetah (especially females with small cubs that from a very early age accompany the female on hunts) to live in closer proximity to their prey than would otherwise be possible.

In this regard, it has been found that organosulfur compounds derived from a protein-rich diet could be a cue by which prey can distinguish a potential predator (Epple et al., 1993; Nolte et al., 1994). If, as mentioned above, sulfur is at the same time used as a territorial-marking substance, it would imply that the cheetah must be equipped to detect sulfur much better than do their predators. With the limited information available from bioassays with cheetah in captivity, there is no proof that this could be the case. Ideally, these possibilities should be tested by investigating the responses of male and female cheetah, other carnivores, and typical prey species to cheetah urine and sulfur in various combinations under completely natural conditions in the wild. This was impossible in the present investigation.

It is not known which, and to what extent, urine constituents play a role in the sexual and territorial behavior of the cheetah, and there is no positive control that could have been used in experiments to determine the reaction of cheetah and other carnivores to cheetah urine and sulfur. In some exploratory experiments that were nevertheless done [with four male and three female cheetah at the Spier facility, as well as breeding pairs of wild cheetah, lion (P. leo), Bengal tigers (P.tigris), caracal (F. caracal), African wild cats (F. silvestris lybica), and wild dogs (L. pictus), at the Tygerberg Zoo], sulfur and cheetah urine elicited practically no response either in cheetah or in the other carnivores. Other odorants, especially benzeneethanethiol, elicited stronger responses than male cheetah urine or sulfur. In an environment that must be saturated with semiochemical messages, no significance can be attached to the results of these bioassays as far as the possible role of sulfur as a component of a territorial-marking pheromone is concerned.

On the other hand, our hypothesis - that the conversion of sulfur-containing compounds in their diet to elemental sulfur enables cheetah to operate as if "invisible" to the olfactory world of their predators as well as their preywas not refuted by the results of these bioassays. The secretion of elemental sulfur by a carabid beetle (Meinwald and Attygalle, personal communication) is the only other known example of the production of elemental sulfur by an animal. It is possible that sulfur is not so rare in animal excretions and secretions, but that it has been overlooked in the urine and/or feces of other species, as it is not detected by FID. It is hoped that biologists will be motivated by the discovery of sulfur in the urine of the cheetah to look for it in other biological material and to consider its possible role in other biological systems.

\section{Acknowledgments}

We thank the Cheetah Outreach Facility for permission to collect urine from cheetah and to conduct bioassays with the animals, Tygerberg Zoological Garden for permission to conduct bioassays with cheetah and several other carnivore species, and the Cheetah Conservation Fund for cheetah urine. Stellenbosch University and the National Research Foundation, Pretoria, South Africa, funded the research. 


\section{Figures}

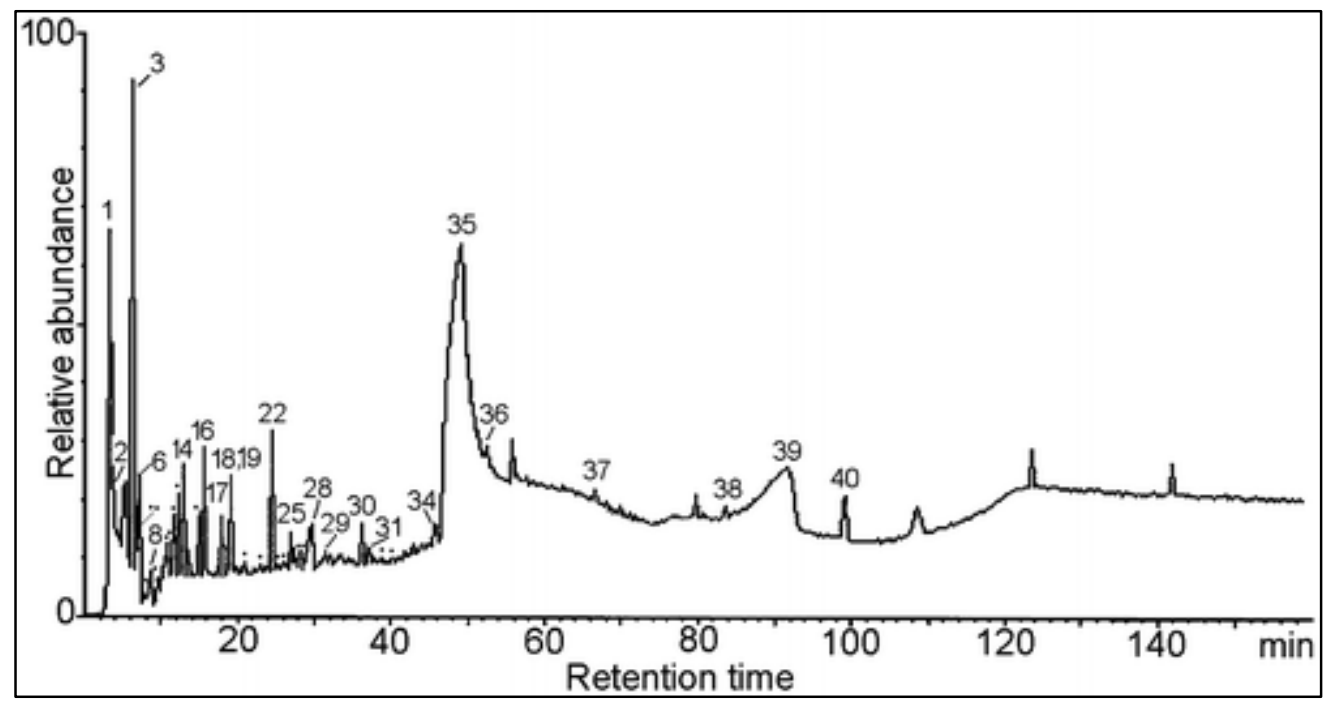

Fig. $1 \mathrm{TIC}$ of volatile constituents extracted for $69 \mathrm{hr}$ at $40^{\circ} \mathrm{C}$ from the headspace vapor of the urine of a female cheetah with a device in which $140 \mu \mathrm{l}$ of PDMS rubber was used, desorbed for $5 \mathrm{~min}$ at $220^{\circ} \mathrm{C}$ in the injector of the GC-MS system, and analyzed on a $40 \mathrm{~m} \times 0.3 \mathrm{~mm}$ glass capillary column coated with PS-089 (DB-5 equivalent) by using a temperature programming rate of $2^{\circ} \mathrm{C} / \mathrm{min}$ from $40^{\circ} \mathrm{C}$ to $280^{\circ} \mathrm{C}$. 
Table

Table 1

Constituents Identified in the Headspace Vapor of Cheetah Urine ${ }^{a}$

\begin{tabular}{|c|c|c|c|}
\hline No. in Figure $\underline{1}$ & Constituent & $\sigma^{7}$ & ? \\
\hline 1 & 2-Butanone & $\sqrt{ }$ & $\sqrt{ }$ \\
\hline 2 & Ethyl propyl ether & & $\sqrt{ }$ \\
\hline 3 & 2-Pentanone & $\sqrt{ }$ & $\checkmark$ \\
\hline 4 & Dipropyl ether & & $\sqrt{ }$ \\
\hline 5 & 3-Pentanone & & $\sqrt{ }$ \\
\hline 6 & Butyl ethyl ether & & $\checkmark$ \\
\hline 7 & Heptane & $\sqrt{ }$ & $\sqrt{ }$ \\
\hline 8 & 1,2-Diethoxyethane & & $\sqrt{ }$ \\
\hline 9 & Dimethyl disulfide & $\sqrt{ }$ & $\sqrt{ }$ \\
\hline 10 & 3-Hexanone & $\checkmark$ & $\checkmark$ \\
\hline 11 & 2-Hexanone & $\sqrt{ }$ & $\sqrt{ }$ \\
\hline 12 & Butyl propyl ether & 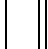 & $\sqrt{ }$ \\
\hline 13 & Hexanal & $\sqrt{ }$ & $\sqrt{ }$ \\
\hline 14 & Ethyl pentyl ether & & $\sqrt{ }$ \\
\hline 15 & 2-Acetylfuran & $\checkmark$ & 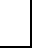 \\
\hline 16 & 3-Methylcyclopentanone & $\checkmark$ & $\sqrt{ }$ \\
\hline 17 & 4-Heptanone & 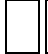 & $\checkmark$ \\
\hline 18 & Cyclohexanone & $\sqrt{ }$ & $\sqrt{ }$ \\
\hline 19 & 2-Heptanone & $\sqrt{ }$ & $\sqrt{ }$ \\
\hline 20 & Dimethyl sulfone & $\checkmark$ & $\checkmark$ \\
\hline 21 & (E)-3-Hepten-2-one & $\checkmark$ & $\sqrt{ }$ \\
\hline 22 & Ethyl-3-methylcyclopentane & $\checkmark$ & $\sqrt{ }$ \\
\hline 23 & Benzaldehyde & $\sqrt{ }$ & $\sqrt{ }$ \\
\hline 24 & 2,4-Heptanedione & 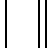 & $\sqrt{ }$ \\
\hline 25 & Phenol & $\sqrt{ }$ & $\sqrt{ }$ \\
\hline 26 & 2-Octanone & | & $\checkmark$ \\
\hline 27 & Octanal & $\checkmark$ & $\checkmark$ \\
\hline 28 & Decane & 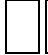 & $\checkmark$ \\
\hline 29 & Acetophenone & $\checkmark$ & $\sqrt{ }$ \\
\hline 30 & 2-Nonanone & 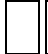 & $\checkmark$ \\
\hline 31 & Nonanal & $\sqrt{ }$ & $\sqrt{ }$ \\
\hline 32 & Phenylacetone & $\checkmark$ & $\checkmark$ \\
\hline 33 & (E)-3-Nonen-2-one & $\checkmark$ & \\
\hline 34 & Decanal & $\checkmark$ & $\sqrt{ }$ \\
\hline 35 & Urea & $\checkmark$ & $\sqrt{ }$ \\
\hline 36 & 2-Undecanone & $\sqrt{ }$ & $\sqrt{ }$ \\
\hline 37 & Sulfur S6 & $\sqrt{ }$ & $\sqrt{ }$ \\
\hline 38 & Myristic acid (tetradecanoic acid) & $\checkmark$ & $\checkmark$ \\
\hline 39 & Cyanuric acid (2,4,6-Trihydroxy-1,3,5-triazine $)^{b}$ & $\checkmark$ & \\
\hline 40 & Sulfur $S_{8}$ & $\sqrt{ }$ & $\sqrt{ }$ \\
\hline
\end{tabular}

arranged in order of elution from an apolar column.

${ }^{b}$ Formed from urea in the injector and on the column of the GC-MS instrument. 


\section{References}

1. Burger, B. V. 2005. Mammalian semiochemicals. Top. Curr. Chem. 240:231-278.

2. Caro, T. M. 1994. Cheetah of the Serengeti Plains: Group Living in Asocial Species. University of Chicago Press, Chicago.

3. Eaton, R. L. 1974. The Cheetah. The Biology, Ecology, and Behavior of an Endangered Species. Van Nostrand Reinhold, New York.

4. Epple, G., Mason, J. R., Nolte, D. L., and Campbell, D. L. 1993. Effects of predator odors on feeding in the mountain beaver (Aplodontia rufa). J. Mammal. 74:715-722.

5. Macdonald, D. 1984. The Encyclopedia of Mammals: 1. Allen \& Unwin, London.

6. Nolte, D. L., Mason, J. R., Epple, G., Aronov, E., and Campbell, D. L. 1994. Why are predator urines aversive to prey? J. Chem. Ecol. 20:1505-1516. 